\title{
Association between Neutrophil Percentage-to-Albumin Ratio and All-Cause Mortality in Critically Ill Patients with Coronary Artery Disease
}

\author{
Tienan Sun $\mathbb{D}^{\mathbb{D}},{ }^{1}$ Hua Shen $\mathbb{D},{ }^{1}$ Qianyun Guo, ${ }^{1}$ Jiaqi Yang, ${ }^{2}$ Guangyao Zhai $\mathbb{D},{ }^{1}$ Jingrui Zhang, $^{1}$ \\ Biyang Zhang $\mathbb{D}^{1}$, Yaodong Ding $\mathbb{D}^{1}{ }^{1}$ Chenghui Cai $\mathbb{D}^{1},{ }^{1}$ and Yujie Zhou $\mathbb{D}^{1}$ \\ ${ }^{1}$ Beijing Anzhen Hospital Affiliated to Capital Medical University, Beijing, China \\ ${ }^{2}$ Beijing Institute of Heart, Lung and Blood Vessel Disease, Beijing, China \\ Correspondence should be addressed to Yujie Zhou; azzyj12@163.com
}

Received 25 June 2020; Revised 31 July 2020; Accepted 13 August 2020; Published 31 August 2020

Academic Editor: Roberto Cangemi

Copyright (C) 2020 Tienan Sun et al. This is an open access article distributed under the Creative Commons Attribution License, which permits unrestricted use, distribution, and reproduction in any medium, provided the original work is properly cited.

\begin{abstract}
Background. Neutrophil percentage-to-albumin ratio (NPAR) has been proved to be associated with clinical outcome of many diseases. This study was aimed at exploring the independent effect of NPAR on all-cause mortality of critically ill patients with coronary artery disease (CAD). Method. NPAR was calculated as neutrophil percentage numerator divided by serum albumin concentration. Clinical endpoints were 30-day, 90-day, and 365-day all-cause mortality. Multivariable Cox proportional hazard models were performed to confirm the association between NPAR and all-cause mortality. Result. 3106 patients with CAD were enrolled. All-cause mortality rates of 30 days $(P<0.001)$, 90 days $(P<0.001)$, and 365 days $(P<0.001)$ increased as NPAR tertiles increased. And after adjusting for possible confounding variables, NPAR was still independently associated with 30-day (third tertile group versus first tertile group: HR, 95\% CI: 1.924, 1.471-2.516; $P$ for trend $<0.001$ ), 90-day (third tertile group versus first tertile group: HR, 95\% CI: 2.053, 1.646-2.560; $P$ for trend $<0.001$ ), and 365-day (third tertile group versus first tertile group: HR, 95\% CI: 2.063, 1.717-2.480; $P$ for trend $<0.001$ ) all-cause mortality in patients with CAD. Subgroup analysis did not find obvious interaction in most subgroups. Conclusion. NPAR was independently correlated with 30-day, 60-day, and 365-day all-cause mortality in critically ill patients with CAD.
\end{abstract}

\section{Introduction}

Although tremendous advances have been made in clinical and basic cardiovascular research over the past few decades, CAD still remains the major cause of death all over the world $[1,2]$; readily accessible and inexpensive prognostic predictors are still necessary for patients with CAD, especially for critically ill patients.

Inflammation was proved to be related to atherosclerosis closely and played a key role in coronary plaque progression and adverse events after stent implantation [3-5]. Neutrophil, as one of classic cellular effectors, plays an important role in mediating inflammatory responses $[3,4]$. Serum albumin concentration, as a classic measure of state of nutrition, is also effected by inflammation [6]. Lower serum albumin concentration was also proved to have a close connection to bad clinical outcomes in patients with CAD mainly due to malnutrition and inflammation $[7,8]$. The pathophysiological changes caused by low serum albumin concentration also contributed to the development of cardiovascular diseases.

As a combination of two classical clinical evaluation parameters, NPAR is calculated as neutrophil percentage numerator divided by serum albumin concentration; a higher NPAR can indicate an increase in neutrophil percentage and a decrease in serum albumin concentration. Moreover, by the calculation of NPAR, the changes of these two indicators are amplified; especially in some cases, clinicians often ignore the significance of these two indicators, for example, when the neutrophil ratio is high and the albumin is low, but both are within the normal range. Previous studies also showed that a higher NPAR was associated with clinical outcomes 
of many diseases such as severe sepsis and acute kidney injury $[9,10]$. In patients with ST-segment elevation myocardial infarction (STEMI), a higher NPAR was related to higher rates of death and reinfarction during hospitalization [11]. Based on the above evidence, we deduced that NPAR could influence the mortality of critically ill patients with $\mathrm{CAD}$, and for all we know, there is no study reporting the influence of NPAR on mortality of critically ill patients with CAD.

\section{Method}

2.1. Data Source. We retrieved all data from an openly available critical care database named Medical Information Mart for Intensive Care III (MIMIC-III, version 1.4) [12], which included more than 60000 intensive care unit (ICU) stays and more than 50000 stays for adult patients. The data in MIMIC-III were collected from June 2001 to October 2012 in Beth Israel Deaconess Medical Center, including general information (patient demographics, birth and death, ICU admission, and discharge information), vital signs, laboratory data, the balance of body fluid, reports, medication, and nursing record. Protecting Human Research Participants exam was passed to gain access to the MIMIC-III database, and our certificate number is 9027152 .

2.2. Study Population and Definition of NPAR. The selected population must meet the following requirements: (1) adult patients (age $\geq 18$ ) who were diagnosed with coronary atherosclerosis or myocardial infarction according to ICD-9 diagnosis code and (2) parameters of neutrophil percent and serum albumin concentration were available after admission to ICU. Patients meeting the following criteria were excluded: (1) patients were under 18 years old and (2) lacking neutrophil percentage or serum albumin concentration data during their stay in the intensive care unit. NPAR was calculated as neutrophil percentage numerator divided by serum albumin concentration. Neutrophil percentage and serum albumin concentration were obtained by the first blood test after admission to the ICU and measured at the same time.

2.3. Data Extraction. Structure query language (SQL) was applied to collect patient information. Demographics, comorbidities and medical history, laboratory parameters, medication, scoring system, vital signs, and survival information were extracted. Demographics included age, gender, and ethnicity. Vital signs included heart beat (HR), systolic blood pressure (SBP), diastolic blood pressure (DBP), and mean blood pressure (MBP). Comorbidities and medical history included atrial fibrillation (AF), hypertension, chronic obstructive pulmonary disease (COPD), diabetes, hypercholesterolemia, congestive heart failure (CHF), prior myocardial infarction (prior MI), and prior stroke. Laboratory parameters included neutrophil, albumin, creatinine, activated partial thromboplastin time (APTT), white blood cell (WBC), hemoglobin, hematocrit, platelet, glucose, lowdensity lipoprotein cholesterol (LDL-C), troponin T, blood urea nitrogen (BUN), sodium, potassium, high-density lipo- protein cholesterol (HDL-C), international normalization ratio (INR), total cholesterol (TC), alanine aminotransferase (ALT), prothrombin time (PT), and C-reactive protein (CRP). Medication included beta-blockers, aspirin, thienopyridines, oral anticoagulants, angiotensin-converting enzyme inhibitors (ACEIs), angiotensin receptor blockers (ARBs), and statins. Scoring system included sequential organ failure assessment score (SOFA) [13] and simplified acute physiology score II (SAPS II) [14]. All the laboratory parameters were collected within 48 hours after admission to the ICU. Vital signs were extracted from a table named "vitalsfirstday" of the MIMIC-III database. Comorbidities and medical history were extracted from a table named "diagnoses_icd" of the MIMIC-III database. Laboratory parameters were extracted from a table named "labevents" of the MIMIC-III database. Medication use was extracted from a table named "prescriptions" of the MIMIC-III database. SOFA and SAPS II were extracted from a table named "sofa" and "sapsii" of the MIMIC-III database.

The endpoints of the study were 30-day, 90-day, and 365day all-cause mortality. Survival information was extracted from a table named "patients" of the MIMIC-III database.

2.4. Statistical Analysis. All the patients with CAD were stratified according to NPAR tertiles. All continuous variables in this study were nonnormally distributed, and they were presented as median and interquartile range (IQR). Categorical data was summarized as number and percentage. KruskalWallis or chi-square test was performed to evaluate statistical differences among different groups of NPAR.

Survival rates of different groups were compared by Logrank tests, and the Kaplan-Meier curves were built. Multivariable Cox proportional hazard models were developed to evaluate the independent effect of NAPR on 30-day, 90day, and 365-day all-cause mortality. The first tertile group of NPAR served as the reference group, and the results were summarized as hazard ratios (HRs) with 95\% confidence intervals (CIs). Variables with $P<0.05$ in the univariate analysis and cardiovascular risk factors were included into the multivariate Cox proportional hazard models. In model I, age, gender, and ethnicity were incorporated into adjustment. In model II, age, ethnicity, gender, length of ICU stay (ICU LOS), body mass index (BMI), SBP, DBP, HR, $\mathrm{AMI}$, diabetes, hypercholesterolemia, prior $\mathrm{MI}, \mathrm{CHF}, \mathrm{AF}$, hypertension, beta-blockers, oral anticoagulants, ACEIs, ARBs, BUN, glucose, platelet, WBC, ALT, INR, LDL-C, HDL-C, TC, troponin T, CRP, SOFA, and SAPS II were incorporated into the model. Subgroup analysis was used to determine the influence of NPAR on 30-day all-cause mortality in different subgroups, and $P$ for interaction was calculated.

Receiver operating characteristic (ROC) curve was applied to evaluate the sensitivity and specificity of NPAR. DeLong test was applied to compare the area under the curves (AUC) of different parameters.

A $P$ value of $<0.05$ was considered to be statistically significant. MedCalc and SPSS 23.0 (IBM Corporation) were used to conduct statistical analysis. GraphPad Prism was used to draw Kaplan-Meier curves and ROC curves. 


\section{Result}

3.1. Patient Characteristics. 3106 critically ill patients with CAD were enrolled in this study (Figure 1), whose characteristics stratified by NPAR tertiles were recorded. 1023 patients were included in the first tertile group (NPAR $<22.1$ ), 1058 patients were included in second tertile group $(22.1 \leq$ NPAR $<27.9)$, and 1025 patients were included in third tertile group (NPAR $\geq 27.9$ ). As displayed in Table 1, the median (IQR) of the NPAR level was 24.7 (20.8-30.0). 1975 men and 1131 women were included, most of whom were white. Patients in the highest tertile of the NPAR level presented more comorbidities or history of hypercholesterolemia, $\mathrm{CHF}, \mathrm{AF}, \mathrm{COPD}$, prior $\mathrm{MI}$, and prior stroke and less comorbidities of hypertension and diabetes. Moreover, patients in the highest tertile of the NPAR level received less thienopyridines, oral anticoagulants, beta-blockers, ACEIs, ARBs, and statin treatment. They also had higher $H R$, WBC, platelet, creatinine, BUN, sodium, PT, APTT, INR, CRP, SOFA, and SAPS II but lower BMI, SBP, DBP, MBP, hemoglobin, LDL-C, HDL-C, and TC.

3.2. NPAR and Outcomes. As shown in Table 2, the overall in-hospital, 30-day, 90-day, and 365-day all-cause mortality was $15.9 \%, 17.9 \%, 25.5 \%$, and $34.6 \%$, respectively. The rates of in-hospital, 30-day, 90-day, and 365-day all-cause mortality were all significantly increased as the NPAR tertiles increased. Moreover, ICU LOS significantly increased in the higher NPAR group.

The survival curves of 30-day (Log rank, $P<0.001$ ), 90day (Log rank, $P<0.001$ ), and 365-day (Log rank, $P<0.001)$ all-cause mortality stratified by the tertiles of NPAR are shown in Figure 2, which demonstrated significantly lower cumulative survivals with higher NPAR tertiles. The independent effect of NAPR on all-cause mortality was confirmed by Cox regression models. In model I, age, ethnicity, and gender, were incorporated into the regression model; compared with the first tertile, the highest 30-day (third tertile group versus first tertile group: HR, 95\% CI: 3.387, 2.6354.353; $P$ for trend $<0.001$ ), 90-day (third tertile group versus first tertile group: HR, 95\% CI: 3.485, 2.831-4.290; $P$ for trend $<0.001$ ), and 365-day (third tertile group versus first tertile group: HR, 95\% CI: 3.315, 2.789-3.940; $P$ for trend $<0.001$ ) all-cause mortality was confirmed in the highest tertile of NPAR. When examined as continuous variables in model I, each unit's higher NPAR was associated with increased 30day (HR, 95\% CI: 1.051, 1.042-1.060; $P<0.001)$, 90-day (HR, 95\% CI: 1.054, 1.046-1.061; $P<0.001$ ), and 365-day (HR, 95\% CI: 1.053, 1.046-1.060; $P<0.001$ ) all-cause mortality. In model II, age, ethnicity, gender, length of ICU stay (ICU LOS), body mass index (BMI), SBP, DBP, HR, AMI, diabetes, hypercholesterolemia, prior $\mathrm{MI}, \mathrm{CHF}, \mathrm{AF}$, hypertension, beta-blockers, oral anticoagulants, ACEIs, ARBs, BUN, glucose, platelet, WBC, ALT, INR, LDL-C, HDL-C, TC, troponin T, CRP, SOFA, and SAPS II were incorporated. NPAR was still independently associated with 30-day (third tertile group versus first tertile group: HR, 95\% CI: 1.924 , 1.471-2.516; $P$ for trend < 0.001), 90-day (third tertile group versus first tertile group: $\mathrm{HR}, 95 \% \mathrm{CI}$ : 2.053, 1.646-2.560; $P$

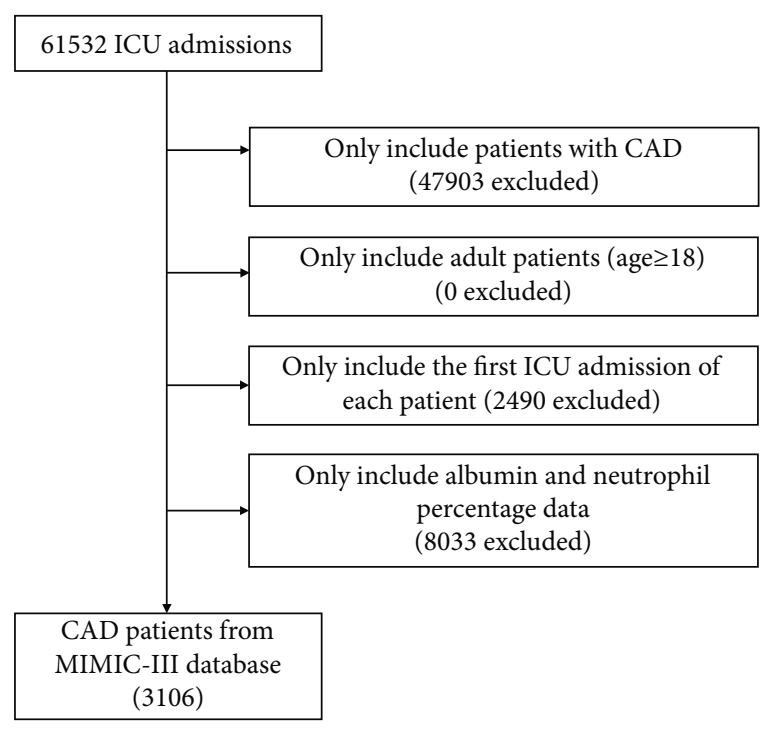

FIGURE 1: Flow chart of study population. ICU: intensive care unit; CAD: coronary artery disease.

for trend $<0.001$ ), and 365-day (third tertile group versus first tertile group: HR, 95\% CI: 2.063, 1.717-2.480; $P$ for trend $<0.001)$ all-cause mortality in patients with CAD. When examined as continuous variables in model II, each unit's higher NPAR was still associated with increased 30-day (HR, 95\% CI: 1.032, 1.021-1.043; $P<0.001$ ), 90-day (HR, 95\% CI: $1.032,1.023-1.040 ; P<0.001$ ), and 365-day (HR, 95\% CI: $1.032,1.024-1.040 ; P<0.001)$ all-cause mortality independently (Table 3 ).

The ability to predict all-cause mortality of NPAR is presented in Figure 3. The AUCs of NPAR for 30-day, 90-day, and 365-day mortality were 0.6758, 0.6871, and 0.6892, respectively. Comparing AUCs, the ability to predict the 30-day mortality of NPAR was proved to be lower than that of SAPS II $(P=0.009)$ but better than that of neutrophil percentage $(P<0.0001)$ alone. Comparing with the SOFA score $(P=0.7254)$ and albumin $(P=0.6521)$, no significant difference was found.

3.3. Subgroup Analysis. In most subgroups, no obvious interaction was observed. But increased risk of 30-day all-cause mortality was observed in patients with SAPS II score $<39$ and BUN $<25$ ( $P$ for interaction $=0.002,0.003)($ Table 4$)$.

\section{Discussion}

This study showed that NPAR was an independent risk factor of 30-day, 90-day, and 365-day all-cause mortality in patients with CAD, even after adjusting for possible confounding variables. ROC curves revealed that NPAR had a moderate ability to predict all-cause mortality of critically ill patients with CAD. From the subgroup analysis, we did not find obvious interaction in most subgroups.

Inflammation was associated with atherosclerosis closely and played an important role in coronary plaque progression and adverse events after stent implantation [3-5]. A previous study showed that reducing classical inflammatory cascade 
TABLE 1: Characteristics of study patients by NPAR tertiles.

\begin{tabular}{|c|c|c|c|c|c|}
\hline Characteristics & Total $(n=3106)$ & Tertile $1(n=1023)<22.1$ & $\begin{array}{c}\text { Tertiles of NPAR } \\
\text { Tertile } 2(n=1058) \\
\quad \geq 22.1,<27.9\end{array}$ & Tertile $3(n=1025) \geq 27.9$ & $P$ value \\
\hline Age (years) & $72.5(63.6-81.0)$ & $69.0(60.3-78.0)$ & $73.6(63.9-81.9)$ & $75.0(66.6-82.6)$ & $<0.001$ \\
\hline Gender, $n(\%)$ & & & & & $<0.001$ \\
\hline Male & $1975(63.6)$ & $723(70.7)$ & $644(60.9)$ & $608(59.3)$ & \\
\hline Female & $1131(36.4)$ & $300(29.3)$ & $414(39.1)$ & $417(40.7)$ & \\
\hline Ethnicity, $n(\%)$ & & & & & 0.450 \\
\hline White & $2241(72.2)$ & $727(71.1)$ & $783(74.0)$ & $731(71.3)$ & \\
\hline Black & $160(5.2)$ & $50(4.9)$ & $55(5.2)$ & $55(5.4)$ & \\
\hline Other & $705(22.6)$ & $246(24)$ & $220(20.8)$ & $239(23.3)$ & \\
\hline BMI $\left(\mathrm{kg} / \mathrm{m}^{2}\right)$ & $27.4(23.8-31.1)$ & $28.2(24.9-31.8)$ & $27.3(23.6-31.2)$ & $26.6(23.0-30.1)$ & $<0.001$ \\
\hline HR (beats/minute) & $84(74-93)$ & $83(75-91)$ & $83(73-92)$ & $86(75-96)$ & $<0.001$ \\
\hline $\mathrm{SBP}(\mathrm{mmHg})$ & $113(104-123)$ & $114(106-124)$ & $113(105-124)$ & $112(102-122)$ & $<0.001$ \\
\hline DBP (mmHg) & $56(51-63)$ & $58(53-64)$ & $57(51-64)$ & $55(50-62)$ & $<0.001$ \\
\hline $\mathrm{MBP}(\mathrm{mmHg})$ & $74(69-81)$ & $75(71-81)$ & $74(69-81)$ & $73(67-80)$ & $<0.001$ \\
\hline AMI, $n(\%)$ & $93(3.0)$ & $19(1.9)$ & $32(3.0)$ & $42(4.1)$ & 0.012 \\
\hline \multicolumn{6}{|c|}{ Comorbidities and medical history, $n(\%)$} \\
\hline Hypertension & $1476(47.5)$ & $596(58.3)$ & $478(45.2)$ & $402(39.2)$ & $<0.001$ \\
\hline Diabetes & $1220(39.3)$ & $406(39.7)$ & $450(42.5)$ & $364(35.5)$ & 0.004 \\
\hline Hypercholesterolemia & $1258(40.5)$ & $521(50.9)$ & $407(38.5)$ & $330(32.2)$ & $<0.001$ \\
\hline $\mathrm{CHF}$ & $1576(50.7)$ & $420(41.1)$ & $615(58.1)$ & $541(52.8)$ & $<0.001$ \\
\hline $\mathrm{AF}$ & $1273(41.0)$ & $386(37.7)$ & $445(42.1)$ & $442(43.1)$ & 0.031 \\
\hline COPD & $93(3.0)$ & $19(1.9)$ & $32(3.0)$ & $42(4.1)$ & 0.012 \\
\hline Prior MI & $493(15.9)$ & $136(13.3)$ & $180(17.0)$ & $177(17.3)$ & 0.022 \\
\hline Prior stroke & $95(3.1)$ & $22(2.2)$ & $37(3.5)$ & $36(3.5)$ & 0.120 \\
\hline \multicolumn{6}{|l|}{ Laboratory parameters } \\
\hline Neutrophil (\%) & $80.7(72.0-87.0)$ & $70.3(63.0-77.2)$ & $82.1(76.0-87.0)$ & $86.5(81.1-95.0)$ & $<0.001$ \\
\hline Albumin (g/dL) & $3.2(2.7-3.7)$ & $3.8(3.5-4.0)$ & $3.3(3.0-3.5)$ & $2.5(2.3-2.8)$ & $<0.001$ \\
\hline WBC $\left(10^{9} / \mathrm{L}\right)$ & $10.0(7.5-13.0)$ & $9.3(7.0-12.1)$ & $10.0(7.6-13.0)$ & $10.9(7.9-13.9)$ & $<0.001$ \\
\hline Hemoglobin (g/dL) & $10.3(9.3-11.5)$ & $10.6(9.5-11.9)$ & $10.4(9.4-11.5)$ & $10.0(9.1-10.9)$ & $<0.001$ \\
\hline Hematocrit (\%) & $30.7(27.8-34.0)$ & $31.0(27.9-34.9)$ & $31.0(28.2-34.2)$ & $31.0(27.4-32.7)$ & $<0.001$ \\
\hline Platelet $\left(10^{9} / \mathrm{L}\right)$ & $208.0(149.0-289.0)$ & $195.0(146.0-262.0)$ & $207.0(151.0-288.0)$ & $228.0(151.0-316.0)$ & $<0.001$ \\
\hline Glucose (mg/dL) & $123.0(100.0-155.0)$ & $122.0(101.0-151.0)$ & $125.0(102.0-159.0)$ & $121.0(98.0-155.0)$ & 0.077 \\
\hline LDL-C (mg/dL) & $78.0(57.0-107.0)$ & $86.0(64.0-117.0)$ & $77.0(57.0-103.0)$ & $68.0(50.0-97.5)$ & $<0.001$ \\
\hline $\mathrm{HDL}-\mathrm{C}(\mathrm{mg} / \mathrm{dL})$ & $42.0(33.0-51.0)$ & $43.0(36-51)$ & $42.0(33.0-52.0)$ & $39.0(30.0,51.0)$ & 0.001 \\
\hline $\mathrm{TC}(\mathrm{mg} / \mathrm{dL})$ & $148.0(118.0-181.0)$ & $160.0(130.0-192.0)$ & $145.0(118.0-177.0)$ & $134.0(107.0-169.0)$ & $<0.001$ \\
\hline Troponin T (ng/mL) & $0.09(0.02-0.45)$ & $0.07(0.01-0.39)$ & $0.1(0.02-0.53)$ & $0.09(0.02-0.42)$ & 0.001 \\
\hline Creatinine $(\mathrm{mEq} / \mathrm{L})$ & $1.1(0.8-1.7)$ & $1.0(0.8-1.4)$ & $1.1(0.8-1.9)$ & $1.2(0.8-2.1)$ & $<0.001$ \\
\hline BUN (mg/dL) & $25.0(17.0-40.0)$ & $21.0(15.0-30.0)$ & $26.0(18.0-42.0)$ & $29(19.0-48.0)$ & $<0.001$ \\
\hline Sodium (mmol/L) & $138.0(135.0-141.0)$ & $138.0(136.0-140.0)$ & $138.0(135.0-141.0)$ & $139.0(135.0-142.0)$ & $<0.001$ \\
\hline Potassium $(\mathrm{mmol} / \mathrm{L})$ & $4.1(3.8-4.5)$ & $4.2(3.9-4.4)$ & $4.2(3.8-4.4)$ & $4.2(3.8-4.4)$ & 0.469 \\
\hline PT (seconds) & $13.6(12.7-15.2)$ & $13.3(12.5-14.3)$ & $13.8(12.8-15.6)$ & $13.9(12.9-15.6)$ & $<0.001$ \\
\hline APTT (seconds) & $29.8(26.2-38.9)$ & $29.0(25.8-37.7)$ & $30.4(26.4-39.4)$ & $30.0(26.4-39.4)$ & 0.007 \\
\hline INR & $1.2(1.1-1.4)$ & $1.1(1.1-1.3)$ & $1.2(1.1-1.5)$ & $1.2(1.1-1.5)$ & $<0.001$ \\
\hline ALT (U/L) & $26(17-40)$ & $25(17-40)$ & $27(17-42)$ & $29(16-40)$ & 0.140 \\
\hline CRP & $13.0(7.3-23.0)$ & $9.4(5.2-18.4)$ & $11.0(7.0-17.0)$ & $19.5(12.4-29.7)$ & $<0.001$ \\
\hline
\end{tabular}


TABLE 1: Continued.

\begin{tabular}{|c|c|c|c|c|c|}
\hline Characteristics & Total $(n=3106)$ & Tertile $1(n=1023)<22.1$ & $\begin{array}{l}\text { Tertiles of NPAR } \\
\text { Tertile } 2(n=1058) \\
\quad \geq 22.1,<27.9\end{array}$ & Tertile $3(n=1025) \geq 27.9$ & $P$ value \\
\hline \multicolumn{6}{|l|}{ Medication use } \\
\hline Aspirin & $2911(93.7)$ & $957(93.5)$ & $991(93.7)$ & $963(94.0)$ & 0.928 \\
\hline Thienopyridines & $1187(38.2)$ & $399(39.0)$ & $445(42.1)$ & $343(33.5)$ & $<0.001$ \\
\hline Oral anticoagulants & $842(27.1)$ & $283(27.7)$ & $322(30.4)$ & $237(23.1)$ & 0.001 \\
\hline Beta-blockers & $2434(78.4)$ & $886(84.7)$ & $827(78.2)$ & $741(72.3)$ & $<0.001$ \\
\hline ACEIs & $1439(46.3)$ & $545(53.3)$ & $492(46.5)$ & $402(39.2)$ & $<0.001$ \\
\hline ARBs & $238(7.7)$ & $89(8.7)$ & $95(9.0)$ & $54(5.3)$ & 0.002 \\
\hline Statins & $2013(67.7)$ & $803(78.5)$ & $725(68.5)$ & $575(56.1)$ & $<0.001$ \\
\hline \multicolumn{6}{|l|}{ Scoring systems } \\
\hline SOFA & $5(3-7)$ & $4(2-6)$ & $4(3-7)$ & $5(3-8)$ & $<0.001$ \\
\hline SAPS II & $39.0(31.0-49.0)$ & $36.0(29.0-44.5)$ & $40.0(32.0-47.0)$ & $43.0(34.0-52.0)$ & $<0.001$ \\
\hline NPAR & $24.7(20.8-30.0)$ & $19.1(16.8-20.7)$ & $24.7(23.2-26.2)$ & $32.9(30.0-37.4)$ & $<0.001$ \\
\hline
\end{tabular}

Data are described as count (percentage) for categorical variables and median (interquartile range) for continuous variables. BMI: body mass index; HR: heart beat; SBP: systolic blood pressure; DBP: diastolic blood pressure; MBP: mean blood pressure; AMI: acute myocardial infarction; CHF: chronic heart failure; AF: atrial fibrillation; COPD: chronic obstructive pulmonary disease; prior MI: prior myocardial infarction; WBC: white blood cell; LDL-C: low-density lipoprotein cholesterol; HDL-C: high-density lipoprotein cholesterol; TC: total cholesterol; BUN: blood urea nitrogen; PT: prothrombin time; APTT: activated partial thromboplastin time; INR: international normalization ratio; ALT: alanine aminotransferase; CRP: C-reactive protein; ACEIs: angiotensin-converting enzyme inhibitors; ARBs: angiotensin receptor blockers; SOFA: sequential organ failure assessment score [13]; SAPS II: simplified acute physiology score II [14].

TABLE 2: Outcome of the study patients by NPAR tertiles.

\begin{tabular}{lcccrc}
\hline Outcomes & Total $(n=3106)$ & Tertile $1(n=1023)<22.1$ & Tertile $2(n=1058) \geq 22.1,<27.9$ & Tertile $3(n=1025) \geq 27.9$ & $P$ value \\
\hline Mortality, $n(\%)$ & & & & \\
30-day mortality & $577(17.9)$ & $80(7.8)$ & $192(18.1)$ & $285(27.8)$ \\
90-day mortality & $792(25.5)$ & $118(11.5)$ & $272(25.7)$ & $402(39.2)$ & $<0.001$ \\
365-day mortality & $1074(34.6)$ & $176(17.2)$ & $369(34.9)$ & $529(51.6)$ & $<0.001$ \\
In-hospital & $495(15.9)$ & $71(6.9)$ & $175(16.5)$ & $249(24.3)$ & $<0.001$ \\
ICU LOS (days) & $3.29(1.83-7.32)$ & $2.46(1.30-4.33)$ & $3.26(1.83-6.96)$ & $5.08(2.24-11.13)$ & $<0.001$ \\
\hline
\end{tabular}

Data are expressed as count (percentage) for categorical variables and median (interquartile range) for continuous variables. ICU LOS: length of ICU stay.

contributed to reducing CAD-related adverse events [15]. WBC played a vital part in the host's defense against damage. In patients with stable angina pectoris or acute coronary syndrome, increased white blood cells were associated with poorer prognosis $[16,17]$. Neutrophil, as an important component of WBC and one of the classic cellular effectors, played an important role in mediating inflammatory responses [3, 4]. Previous studies also showed that high neutrophil-lymphocyte ratio was related to cardiovascular mortality during hospitalization and poor outcomes in patients with STEMI [18, 19]. Besides, high neutrophillymphocyte ratio may contribute to coronary thrombus in patients with non-ST-element elevation acute myocardial infarction [20].

As a marker of nutritional condition and principal component of colloid osmotic pressure, serum albumin concentration was also effected by inflammation [6]. Previous studies showed that low serum albumin concentration was a strong prognostic marker for many diseases, mainly due to malnutrition and inflammation $[6,21]$. Low serum albu- min concentration was proved to be related to the development of ischemic heart disease and was proved to be an independent predictor of first or recurrent myocardial infarction (MI) [22-24]. Moreover, lower serum albumin concentration was also proved to be connected with worse clinical outcomes in patients with CAD [7, 8]. For patients with STEMI, low serum albumin levels, even within the normal range, could still influence long-term all-cause mortality [7]. A study that enrolled 1316 patients with CAD revealed that decreased albumin could predict a higher rate of allcause death after percutaneous transluminal coronary intervention [8]. And a previous study also explained that why low albumin concentration could affect the outcome of patients with CAD, myocardial edema, and fluid retention played a key part in the progress of disease [23].

As a combination of two classical clinical evaluation parameters, NPAR was proved to be an independent predictor for clinical outcomes of many diseases such as severe sepsis, acute kidney injury, and STEMI [9-11], which had the advantage of simplicity, cheapness, and timeliness. A 


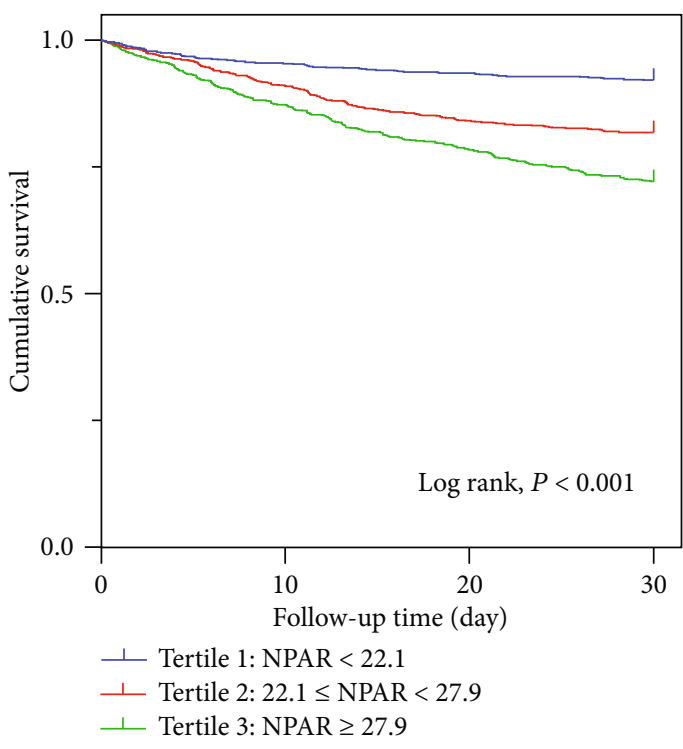

(a)

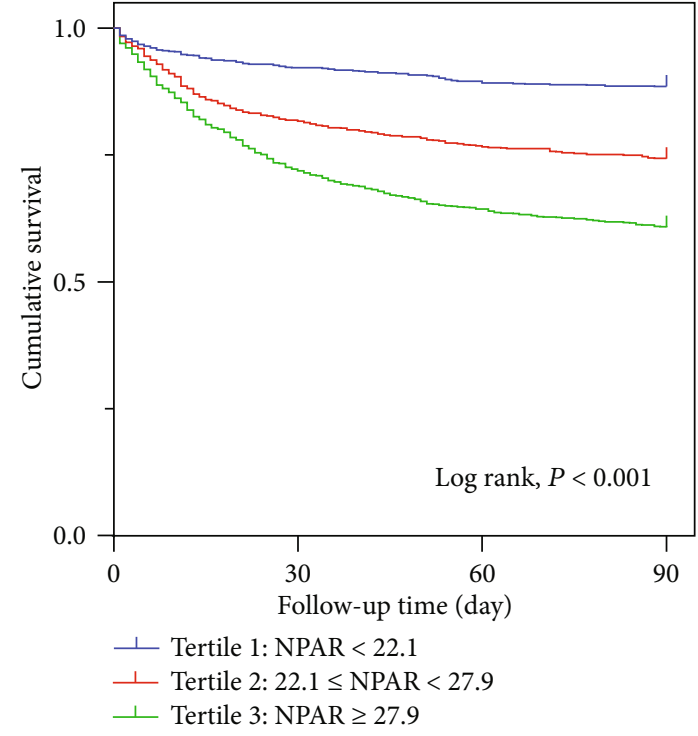

(b)

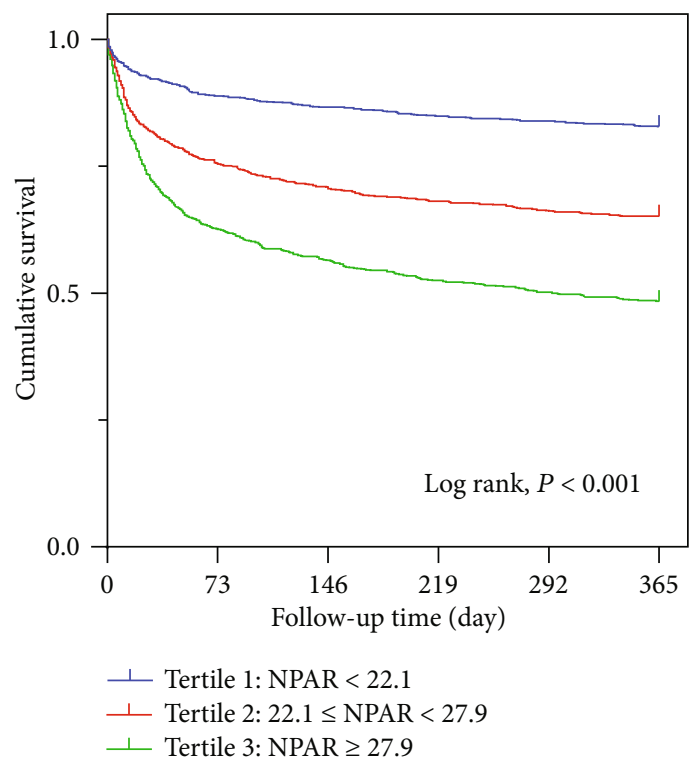

(c)

FIgURE 2: (a) Kaplan-Meier curves showing the association between the NPAR tertiles and 30-day all-cause mortality. (b) Kaplan-Meier curves showing the association between the NPAR tertiles and 90-day all-cause mortality. (c) Kaplan-Meier curves showing the association between the NPAR tertiles and 365-day all-cause mortality.

recent study demonstrated that a higher NPAR was related to higher rates of death and reinfarction during hospitalization in patients with STEMI [11]. Our study draw a similar conclusion that NPAR was an independent marker for allcause mortality and had a moderate ability to predict allcause mortality of critically ill patients with CAD. Although both neutrophil percentage and albumin could influence the outcomes of patients with CAD, NPAR may offer more predictive power from ROC curves. Moreover, comparing AUCs between NPAR and SOFA score, no significant difference was observed; although it was rash to think that NPAR was as effective as SOFA, at least this reminded us that when SOFA score cannot be calculated in some special circum- stances, NPAR may be able to provide guidance for our clinical work. ROC curves also showed that the ability to predict 30-day mortality of NPAR was proved to be lower than that of SAPS II; as mentioned above, it was unrealistic for NPAR to achieve the same effectiveness as traditional classic scores, but considering the complexity of the calculation of SAPS II, NPAR had certain advantages in terms of simplicity. Given the low cost, availability, and capacity to predict mortality, NPAR is clinically valuable for critically ill patients with CAD. Especially in some special cases, such as remote areas with underdeveloped medical services or when patients are unable to perform more complex tests, NPAR may alert the clinicians. 


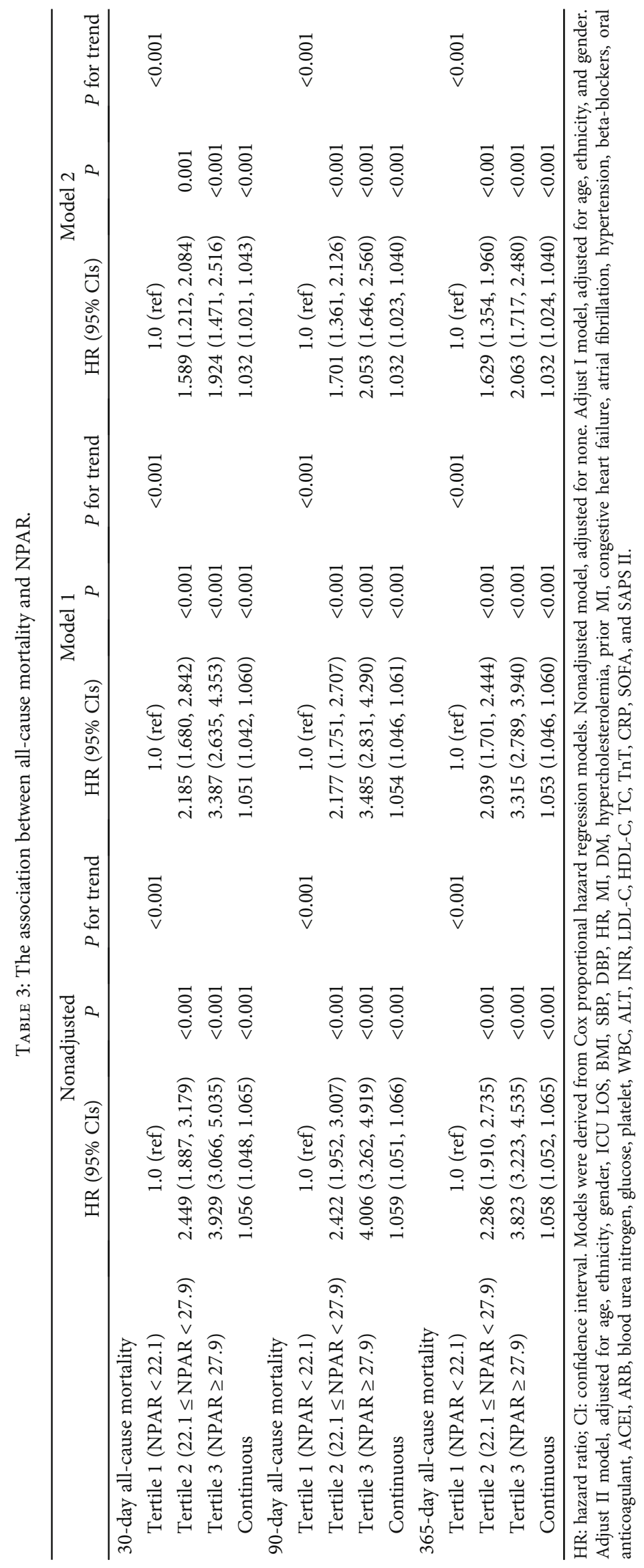




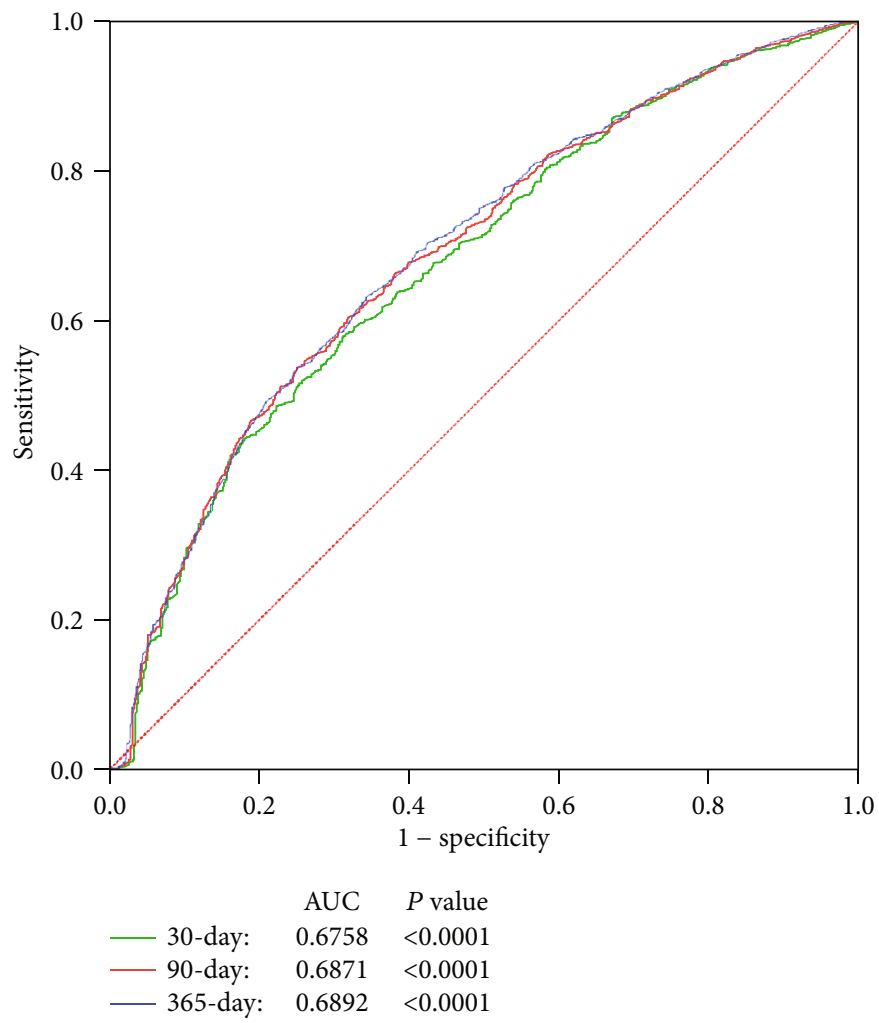

(a)

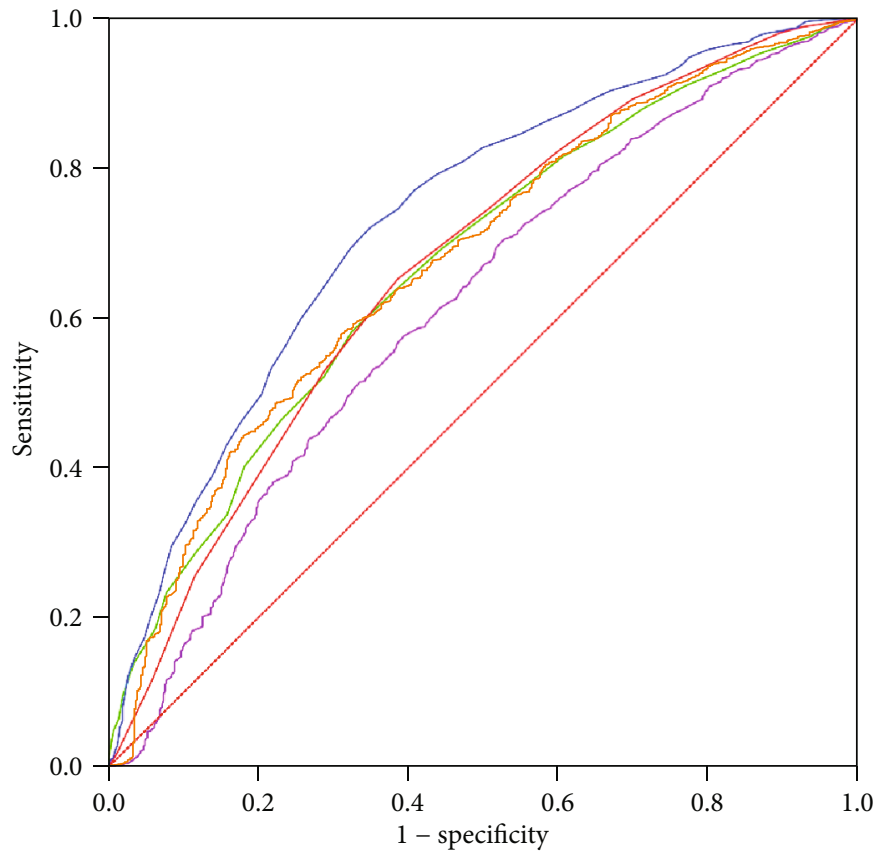

$\begin{array}{lcc} & \text { AUC } & P \text { value } \\ \text { NPAR: } & 0.6758 & <0.0001 \\ \text { Neutrophil: } & 0.6151 & <0.0001 \\ \text { Albumin: } & 0.6717 & <0.0001 \\ \text { - SOFA: } & 0.6694 & <0.0001 \\ \text { SAPS II: } & 0.7322 & <0.0001\end{array}$

(b)

FIGURE 3: (a) ROC curves for the prediction of 30-day, 90-day, and 365-day all-cause mortality. (b) ROC curves for the prediction of 30-day all-cause mortality of NPAR, neutrophil percentage, serum albumin concentration, SOFA score, and SAPS II score. 
TABLE 4: Subgroup analysis of associations between 30-day all-cause mortality and NPAR.

\begin{tabular}{|c|c|c|c|c|c|}
\hline & $N$ & NPAR $<22.1$ (reference) & $\begin{array}{c}22.1 \leq \mathrm{NPAR}<27.9 \\
\text { HR }(95 \% \text { CIs })\end{array}$ & $\begin{array}{l}\text { NPAR } \geq 27.9 \\
\text { HR (95\% CIs) }\end{array}$ & $P$ for interaction \\
\hline AMI & & & & & 0.886 \\
\hline No & 3013 & 1.0 (ref) & $2.480(1.904,3.230)$ & $3.951(3.072,5.082)$ & \\
\hline Yes & 93 & 1.0 (ref) & $1.450(0.281,7.477)$ & $2.878(0.644,12.863)$ & \\
\hline $\mathrm{CHF}$ & & & & & 0.862 \\
\hline No & 1530 & 1.0 (ref) & $2.946(1.956,4.438)$ & $4.618(3.149,6.771)$ & \\
\hline Yes & 1576 & 1.0 (ref) & $1.899(1.353,2.666)$ & $3.172(2.290,4.393)$ & \\
\hline $\mathrm{AF}$ & & & & & 0.912 \\
\hline No & 1833 & 1.0 (ref) & $2.069(1.470,2.912)$ & $3.594(2.610,4.949)$ & \\
\hline Yes & 1273 & 1.0 (ref) & $3.013(2.000,4.540)$ & $4.398(2.960,6.535)$ & \\
\hline Hypertension & & & & & 0.066 \\
\hline No & 1630 & 1.0 (ref) & $2.197(1.558,3.099)$ & $3.169(2.281,4.403)$ & \\
\hline Yes & 1476 & 1.0 (ref) & $2.459(1.641,3.682)$ & $4.528(3.094,6.629)$ & \\
\hline Diabetes & & & & & 0.934 \\
\hline No & 1886 & 1.0 (ref) & $2.585(1.839,3.632)$ & $4.044(2.936,5.571)$ & \\
\hline Yes & 1220 & 1.0 (ref) & $2.269(1.511,3.406)$ & $3.757(2.534,5.568)$ & \\
\hline Hypercholesterolemia & & & & & 0.149 \\
\hline No & 1848 & 1.0 (ref) & $2.190(1.572,3.051)$ & $3.324(2.426,4.555)$ & \\
\hline Yes & 1258 & 1.0 (ref) & $2.635(1.722,4.032)$ & $4.573(3.043,6.872)$ & \\
\hline Prior MI & & & & & 0.428 \\
\hline No & 2613 & 1.0 (ref) & $2.428(1.822,3.236)$ & $4.020(3.064,5.276)$ & \\
\hline Yes & 493 & 1.0 (ref) & $2.412(1.288,4.518)$ & $3.371(1.835,6.190)$ & \\
\hline Beta-blockers & & & & & 0.700 \\
\hline No & 672 & 1.0 (ref) & $2.722(1.639,4.520)$ & $3.715(2.283,6.046)$ & \\
\hline Yes & 2434 & 1.0 (ref) & $2.183(1.605,2.968)$ & $3.590(2.682,4.806)$ & \\
\hline Oral anticoagulant & & & & & 0.560 \\
\hline No & 2264 & 1.0 (ref) & $2.512(1.895,3.330)$ & $3.744(2.866,4.890)$ & \\
\hline Yes & 842 & 1.0 (ref) & $2.494(1.250,4.976)$ & $4.468(2.288,8.725)$ & \\
\hline ACEIs & & & & & 0.907 \\
\hline No & 1667 & 1.0 (ref) & $2.142(1.567,2.927)$ & $3.473(2.590,4.655)$ & \\
\hline Yes & 1439 & 1.0 (ref) & $2.830(1.761,4.548)$ & $3.649(2.278,5.845)$ & \\
\hline $\mathrm{ARBs}$ & & & & & 0.452 \\
\hline No & 2868 & 1.0 (ref) & $2.482(1.903,3.237)$ & $3.811(2.960,4.907)$ & \\
\hline Yes & 238 & 1.0 (ref) & $1.912(0.478,7.645)$ & $5.299(1.435,19.577)$ & \\
\hline BUN (mg/dL) & & & & & 0.003 \\
\hline$<25$ & 1542 & 1.0 (ref) & $3.535(2.108,5.929)$ & $5.892(3.579,9.701)$ & \\
\hline$\geq 25$ & 1564 & 1.0 (ref) & $1.596(1.180,2.159)$ & $2.358(1.772,3.139)$ & \\
\hline Glucose (mg/dL) & & & & & 0.485 \\
\hline$<123$ & 1540 & 1.0 (ref) & $2.052(1.387,3.037)$ & $3.443(2.393,4.954)$ & \\
\hline$\geq 123$ & 1566 & 1.0 (ref) & $2.767(1.946,3.934)$ & $4.421(3.149,6.209)$ & \\
\hline Platelet $\left(10^{9} / \mathrm{L}\right)$ & & & & & 0.858 \\
\hline$<208$ & 1539 & 1.0 (ref) & $2.287(1.641,3.187)$ & $3.990(2.905,5.480)$ & \\
\hline$\geq 208$ & 1567 & 1.0 (ref) & $2.824(1.844,4.327)$ & $4.321(2.880,6.482)$ & \\
\hline $\operatorname{WBC}\left(10^{9} / \mathrm{L}\right)$ & & & & & 0.134 \\
\hline$<10$ & 1409 & 1.0 (ref) & $2.010(1.357,2.976)$ & $2.797(1.898,4.123)$ & \\
\hline$\geq 10$ & 1697 & 1.0 (ref) & $2.750(1.931,3.917)$ & $4.426(3.167,6.185)$ & \\
\hline $\operatorname{ALT}(\mathrm{U} / \mathrm{L})$ & & & & & 0.436 \\
\hline$<26$ & 1488 & 1.0 (ref) & $2.647(1.793,3.909)$ & $4.349(3.001,6.304)$ & \\
\hline$\geq 26$ & 1618 & 1.0 (ref) & $2.264(1.593,3.216)$ & $3.545(2.540,4.949)$ & \\
\hline
\end{tabular}


TABLe 4: Continued.

\begin{tabular}{|c|c|c|c|c|c|}
\hline & $N$ & NPAR $<22.1$ (reference) & $\begin{array}{c}22.1 \leq \mathrm{NPAR}<27.9 \\
\text { HR }(95 \% \text { CIs })\end{array}$ & $\begin{array}{l}\text { NPAR } \geq 27.9 \\
\text { HR (95\% CIs) }\end{array}$ & $P$ for interaction \\
\hline INR & & & & & 0.046 \\
\hline$<1.2$ & 1272 & 1.0 (ref) & $2.639(1.685,4.133)$ & $4.993(3.289,7.581)$ & \\
\hline$\geq 1.2$ & 1834 & 1.0 (ref) & $2.151(1.559,2.968)$ & $3.178(2.334,4.328)$ & \\
\hline LDL-C (mg/dL) & & & & & 0.380 \\
\hline$<84.1$ & 1539 & 1.0 (ref) & $2.059(1.441,2.942)$ & $3.355(2.402,4.868)$ & \\
\hline$\geq 84.1$ & 1567 & 1.0 (ref) & $2.852(1.946,4.179)$ & $4.365(3.010,6.330)$ & \\
\hline HDL-C (mg/dL) & & & & & 0.732 \\
\hline$<43.5$ & 1552 & 1.0 (ref) & $2.312(1.592,3.356)$ & $3.842(2.711,5.444)$ & \\
\hline$\geq 43.5$ & 1554 & 1.0 (ref) & $2.574(1.787,3.708)$ & $3.915(2.744,5.585)$ & \\
\hline $\mathrm{TC}(\mathrm{mg} / \mathrm{dL})$ & & & & & 0.452 \\
\hline$<153.6$ & 1517 & 1.0 (ref) & $2.458(1.672,3.614)$ & $3.663(2.528,5.307)$ & \\
\hline$\geq 153.6$ & 1589 & 1.0 (ref) & $2.457(1.724,3.503)$ & $4.182(2.995,5.839)$ & \\
\hline Troponin T (ng/mL) & & & & & 0.644 \\
\hline$<0.02$ & 1446 & 1.0 (ref) & $1.960(1.297,2.960)$ & $3.731(2.565,5.427)$ & \\
\hline$\geq 0.02$ & 1660 & 1.0 (ref) & $2.622(1.860,3.697)$ & $3.890(2.789,5.425)$ & \\
\hline CRP (mg/L) & & & & & 0.127 \\
\hline$<13.0$ & 1550 & 1.0 (ref) & $2.791(2.023,3.849)$ & $3.894(2.736,5.541)$ & \\
\hline$\geq 13.0$ & 1556 & 1.0 (ref) & $1.959(1.254,3.061)$ & $4.053(2.732,6.014)$ & \\
\hline SOFA & & & & & 0.099 \\
\hline$<5$ & 1513 & 1.0 (ref) & $2.262(1.408,3.634)$ & $4.510(2.882,7.059)$ & \\
\hline$\geq 5$ & 1593 & 1.0 (ref) & $2.484(1.817,3.396)$ & $3.288(2.441,4.430)$ & \\
\hline SAPS II & & & & & 0.002 \\
\hline$<39$ & 1472 & 1.0 (ref) & $4.189(2.310,7.597)$ & $7.064(3.940,12.662)$ & \\
\hline$\geq 39$ & 1634 & 1.0 (ref) & $1.789(1.337,2.395)$ & $2.477(1.883,3.259)$ & \\
\hline BMI $\left(\mathrm{kg} / \mathrm{m}^{2}\right)$ & & & & & 0.297 \\
\hline$<27.4$ & 1564 & 1.0 (ref) & $2.245(1.578,3.194)$ & $3.412(2.444,4.764)$ & \\
\hline$\geq 27.4$ & 1542 & 1.0 (ref) & $2.579(1.749,3.802)$ & $4.296(2.959,6.235)$ & \\
\hline $\mathrm{SBP}(\mathrm{mmHg})$ & & & & & 0.410 \\
\hline$<113$ & 1547 & 1.0 (ref) & $2.421(1.720,3.408)$ & $3.564(2.574,4.933)$ & \\
\hline$\geq 113$ & 1559 & 1.0 (ref) & $2.421(1.617,3.625)$ & $4.206(2.865,6.174)$ & \\
\hline $\mathrm{DBP}(\mathrm{mmHg})$ & & & & & 0.465 \\
\hline$<57$ & 1561 & 1.0 (ref) & $3.066(2.053,4.579)$ & $4.711(3.211,6.913)$ & \\
\hline$\geq 57$ & 1545 & 1.0 (ref) & $2.201(1.425,2.866)$ & $3.355(2.404,4.682)$ & \\
\hline HR (beats/minute) & & & & & 0.149 \\
\hline$<84$ & 1570 & 1.0 (ref) & $3.182(2.135,4.743)$ & $5.012(3.398,7.393)$ & \\
\hline$\geq 84$ & 1536 & 1.0 (ref) & $1.957(1.382,2.771)$ & $3.155(2.286,4.356)$ & \\
\hline ICU LOS (day) & & & & & 0.004 \\
\hline$<3.3$ & 1554 & 1.0 (ref) & $3.009(2.060,4.397)$ & $5.284(3.641,7.671)$ & \\
\hline$\geq 3.3$ & 1552 & 1.0 (ref) & $1.832(1.279,2.624)$ & $2.709(1.937,3.788)$ & \\
\hline
\end{tabular}

HR: hazard ratio; CI: confidence interval. AMI: acute myocardial infarction; CHF: chronic heart failure; AF: atrial fibrillation; Prior MI: prior myocardial infarction; ACEIs: angiotensin-converting enzyme inhibitors; ARBs: angiotensin receptor blockers; BUN: blood urea nitrogen; WBC: white blood cell; ALT: alanine aminotransferase; INR: international normalization ratio; LDL-C: low-density lipoprotein cholesterol; HDL-C: high-density lipoprotein cholesterol; TC: total cholesterol; CRP: C-reactive protein; SOFA: sequential organ failure assessment score; SAPS II: simplified acute physiology score II; BMI: body mass index; SBP: systolic blood pressure; DBP: diastolic blood pressure; HR: heart beat; ICU LOS: length of ICU stay.

\section{Limitation}

This study was a single retrospective study; inevitable bias may affect the authenticity of the results. In general, the more key variables a model contains, the more accurate its predic- tions will be. But constrained by public database, a lot of information that may affect the model was not collected, like smoking and alcohol. Moreover, neutrophil percentage and serum albumin concentration used in this analysis were obtained by the first blood test after admission to the ICU, 
but given the dynamic nature of these indicators, random errors caused by using only the first blood results were inevitable. The inability to dynamically observe NPAR was also one of the flaws of this study. In addition to this, other important information was also not collected such as specific causes of death, specific coronary artery lesions, type of myocardial infarction, specific clinical symptom, and left ventricular ejection fraction. Moreover, due to lack of albumin data, the sample size of this study declined significantly. In order to verify the conclusion of this study, a prospective casecontrol study may be needed.

\section{Conclusions}

NPAR was independently correlated with 30-day, 90-day, and 365-day all-cause mortality in critically ill patients with CAD. A prospective case-control study was needed to verify this conclusion.

\section{Data Availability}

All data used in this analysis were from an openly available critical care database named MIMIC-III. Protecting Human Research Participants exam was passed to gain access to the MIMIC-III database, and our certificate number is 9027152 .

\section{Conflicts of Interest}

The authors declare that they have no conflicts of interest.

\section{Authors' Contributions}

All coauthors contributed to data collection and processing.

\section{Supplementary Materials}

1. The file named "Original data" was the raw data for all the analysis of this article. 2. The file named "selected population icd9-code" was used to show all icd9_code related to the diagnosis of coronary artery disease. (Supplementary Materials)

\section{References}

[1] GBD 2015 Mortality and Causes of Death Collaborators, "Global, regional, and national life expectancy, all-cause mortality, and cause-specific mortality for 249 causes of death, 1980-2015: a systematic analysis for the Global Burden of Disease Study 2015," The Lancet, vol. 388, no. 10053, pp. 1459-1544, 2016.

[2] D. Mozaffarian, E. J. Benjamin, A. S. Go et al., "Heart disease and stroke statistics-2016 update: a report from the American Heart Association," Circulation, vol. 133, no. 4, pp. e38-360, 2016.

[3] G. K. Hansson, "Inflammation, atherosclerosis, and coronary artery disease," New England Journal of Medicine, vol. 352, no. 16, pp. 1685-1695, 2005.

[4] P. Libby, "Inflammation in atherosclerosis," Arteriosclerosis, Thrombosis, and Vascular Biolog, vol. 32, no. 9, pp. 20452051, 2012.
[5] F. Crea and P. Libby, "Acute coronary syndromes: the way forward from mechanisms to precision treatment," Circulation, vol. 136, no. 12, pp. 1155-1166, 2017.

[6] B. R. Don and G. Kaysen, "Serum albumin: relationship to inflammation and nutrition," Seminars in Dialysis, vol. 17, no. 6, pp. 432-437, 2004.

[7] Y. Plakht, H. Gilutz, and A. Shiyovich, "Decreased admission serum albumin level is an independent predictor of longterm mortality in hospital survivors of acute myocardial infarction. Soroka Acute Myocardial Infarction II (SAMI-II) project," International Journal of Cardiology, vol. 219, pp. 20-24, 2016.

[8] H. Wada, T. Dohi, K. Miyauchi et al., "Impact of serum albumin levels on long-term outcomes in patients undergoing percutaneous coronary intervention," Heart and Vessels, vol. 32, no. 9, pp. 1085-1092, 2017.

[9] Y. Gong, D. Li, B. Cheng, B. Ying, and B. Wang, "Increased neutrophil percentage-to-albumin ratio is associated with allcause mortality in patients with severe sepsis or septic shock," Epidemiology \& Infection, vol. 148, article e87, 2020.

[10] B. Wang, D. Li, B. Cheng, B. Ying, and Y. Gong, "The neutrophil percentage-to-albumin ratio is associated with all-cause mortality in critically ill patients with acute kidney injury," BioMed Research International, vol. 2020, Article ID 5687672, 9 pages, 2020.

[11] H. Cui, X. Ding, W. Li, H. Chen, and H. Li, “The neutrophil percentage to albumin ratio as a new predictor of in-hospital mortality in patients with ST-segment elevation myocardial infarction," Medical Science Monitor, vol. 25, pp. 7845-7852, 2019.

[12] A. E. W. Johnson, T. J. Pollard, L. Shen et al., "MIMIC-III, a freely accessible critical care database," Scientific Data, vol. 3, no. 1, article 160035, 2016.

[13] J. -L. Vincent, R. Moreno, J. Takala et al., "The SOFA (sepsisrelated organ failure assessment) score to describe organ dysfunction/failure. On behalf of the working group on sepsis-related problems of the European Society of Intensive Care Medicine," Intensive Care Medicine, vol. 22, no. 7, pp. 707-710, 1996.

[14] J.-R. Le Gall, S. Lemeshow, and F. Saulnier, "A new simplified acute physiology score (SAPS II) based on a European/North American multicenter study," JAMA: The Journal of the American Medical Association, vol. 270, no. 24, pp. 2957-2963, 1993.

[15] P. Libby, "Interleukin-1 beta as a target for atherosclerosis therapy: biological basis of CANTOS and beyond," Journal of the American College of Cardiology, vol. 70, no. 18, pp. 22782289, 2017.

[16] L. O. de Labry, E. W. Campion, R. J. Glynn, and P. S. Vokonas, "White blood cell count as a predictor of mortality: results over 18 years from the Normative Aging Study," Journal of Clinical Epidemiology, vol. 43, no. 2, pp. 153-157, 1990.

[17] H. S. Gurm, D. L. Bhatt, A. M. Lincoff et al., "Impact of preprocedural white blood cell count on long term mortality after percutaneous coronary intervention: insights from the EPIC, EPILOG, and EPISTENT trials," Heart, vol. 89, no. 10, pp. 1200-1204, 2003.

[18] T. Palmerini, S. J. Brener, R. Mehran et al., "Leukocyte count is a modulating factor for the mortality benefit of bivalirudin in ST-segment-elevation acute myocardial infarction," Circulation: Cardiovascular Interventions, vol. 6, no. 5, pp. 518-526, 2013. 
[19] R. C. Oncel, M. Ucar, M. S. Karakas et al., "Relation of neutrophil-to-lymphocyte ratio with GRACE risk score to inhospital cardiac events in patients with ST-segment elevated myocardial infarction," Clinical and Applied Thrombosis/Hemostasis, vol. 21, no. 4, pp. 383-388, 2014.

[20] M. Yilmaz, E. Tenekecioglu, B. Arslan et al., "White blood cell subtypes and neutrophil-lymphocyte ratio in prediction of coronary thrombus formation in non-ST-segment elevated acute coronary syndrome," Clinical and Applied Thrombosis/Hemostasis, vol. 21, no. 5, pp. 446-452, 2014.

[21] P. Goldwasser and J. Feldman, "Association of serum albumin and mortality risk," Journal of Clinical Epidemiology, vol. 50, no. 6, pp. 693-703, 1997.

[22] L. Djousse, K. J. Rothman, L. A. Cupples, D. Levy, and R. C. Ellison, "Serum albumin and risk of myocardial infarction and all-cause mortality in the Framingham Offspring Study," Circulation, vol. 106, no. 23, pp. 2919-2924, 2002.

[23] J. J. Nelson, D. Liao, A. R. Sharrett et al., "Serum albumin level as a predictor of incident coronary heart disease: the Atherosclerosis Risk in Communities (ARIC) study," American Journal of Epidemiology, vol. 151, no. 5, pp. 468-477, 2000.

[24] Q. Yang, Y. M. He, D. P. Cai, X. J. Yang, and H. F. Xu, "Risk burdens of modifiable risk factors incorporating lipoprotein (a) and low serum albumin concentrations for first incident acute myocardial infarction," Scientific Reports, vol. 6, no. 1, article 35463, 2016. 